\title{
Commentary: Erythema marginatum as an early symptom of hereditary angioedema: case report of 2 newborns
}

\author{
Inmaculada Martinez-Saguer ${ }^{*}$ and Henriette Farkas ${ }^{2}$ \\ 'Hemophilia Center Rhine Main, Germany \\ ${ }^{2}$ Hungarian Angioedema Center, 3rd Department of Internal Medicine, Semmelweis University, Hungary
}

\section{Article Info}

\section{Article Notes}

Received: May 23, 2016

Accepted: July 04, 2016

\section{*Correspondence:}

Dr. Inmaculada Martinez-Saguer, MD,

Hemophilia Center Rhine Main

Hessenring 13a, Building G

D-64546 Mörfelden Walldorf,

Germany

E-mail: inmaculada.martinez@hzrm.de

() 2016 Martinez-Saguer I. This article is distributed under the terms of the Creative Commons Attribution 4.0 International License.

\section{Keywords}

Hereditary angioedema

Erythema marginatum

Prodromal symptoms

Diagnosis

Children
Abbreviations: C1-INH $=$ C1 inhibitor; C1-INH-HAE $=$ Hereditary angioedema due to C1-inhibitor deficiency

\section{Main text}

In the past couple of years, progress has been made in improving the management of hereditary angioedema due to $\mathrm{C} 1$ inhibitor deficiency (C1-INH-HAE). Nevertheless, misdiagnosis and delayed diagnosis of this rare genetic disease, even for decades after the manifestation of first symptoms, is not uncommon and the awareness of C1-INH-HAE is still low, not only in the general public but also among healthcare professionals ${ }^{1,2}$. Our published manuscript "Erythema marginatum as an early symptom of hereditary angioedema: case report of 2 newborns"3 reported on very early manifestation of erythema marginatum in a 3-day old girl with C1-INH-HAE and in another newborn who had erythema marginatum at birth. The manuscript was published with the aim to raise awareness of this disease and to highlight the potential role of prodromal symptoms, not only as a warning of an impending attack of C1-INH-HAE, but also as indicators for a correct and timely diagnosis in patients with yet undiagnosed C1-INH-HAE.

C1-INH-HAE is are rare autosomal dominantly inherited disorder that usually manifests during childhood ${ }^{4,5}$. Symptoms are caused by a deficiency of functional $\mathrm{C} 1$ inhibitor (C1-INH) that results in elevated bradykinin release, and manifest as recurrent episodes of non-pitting edema in various body tissues, including life-threatening swelling of the upper airway which may lead to suffocation ${ }^{4}$. They are often preceded by prodromes occurring minutes to days before the development of angioedematous swelling or even independently of an attack ${ }^{6,7,8}$. Prodromal symptoms are reported by a high number of patients (about $90 \%$ of patients in recent surveys) ${ }^{9}$ and, if interpreted correctly, may enable the patient to predict an impending attack and trigger early intervention. They usually manifest as pruritus, paresthesias, nausea, fatigue, flue-like symptoms, or malaise ${ }^{6,8-11}$. Erythema marginatum, a non-pitting, non-pruritic, serpiginous rash, first described by Osler in $1888^{12}$, is also a commonly reported prodromal symptom that occurs in about $50 \%$ of C1-INH-HAE patients ${ }^{2,7}$. While the exact pathomechanism of erythema marginatum development in C1-INH-HAE is still unclear, current evidence suggests that it is bradykinin-mediated ${ }^{13}$.

The newborns in our manuscript, now 6 and 28 years old, 
experienced widespread erythema marginatum upon and directly after birth, and on several occasion during their lives, sometimes followed by angioedematous attacks, sometimes not. Due to a positive family history and because the treating physician recognized the symptoms as a potential prodrome of an attack, one of the patients was diagnosed with C1-INH-HAE after her first erythema marginatum at the age of 3 days. This diagnosis enabled the patient's family and physician to act immediately upon symptoms of an abdominal attack 4 weeks later and to successfully treat the girl with human pasteurized nanofiltered C1-INH concentrate (Berinert $®$, CSL Behring, Marburg, Germany). Indeed, active screening of all members of families with a history of C1-INH-HAE, including infants and toddlers, is highly recommended by international guidelines ${ }^{14}$. It has to be noted, however, that antigenic and functional C1-INH levels in newborns are $60 \%$ to $70 \%$ lower than maternal levels anyway which might lead to a false diagnosis of C1-INH deficiency. Repeated testing is therefore recommended at a later age $\mathrm{e}^{15,16}$.

The second case presented in our manuscript describes a girl who had experienced recurrent erythema marginatum from the day she was born and suffered recurrent angioedematous attacks from the age of 5. She finally received her diagnosis of C1-INH-HAE at the age of 10 years. Similar cases of patients who had presented with recurrent erythema marginatum since infancy but were not diagnosed with C1-INH-HAE for many years have been reported by Farkas ${ }^{7}$ and Hubiche et $\mathrm{al}^{17}$. Cases of misdiagnosis and delayed diagnosis of C1-INH-HAE are often characterized by a long ordeal of visits to physicians, hospitalizations, wrong or no treatment, and unwarranted medical procedures, and might even end up fatal in cases of untreated attacks of the upper airways ${ }^{1,2,4,18,19}$. In the 2 cases we presented, C1-INH-HAE attacks were disabling albeit not life-threatening. Although the risk of fatal attacks in children is low $^{20}$ it must not be neglected. Potentially life-threatening laryngeal attacks have been reported as an initial symptom of C1-INH-HAE not only for adults but also for children ${ }^{21,22}$ and there is a report of one such attack that led to death due to asphyxiation in a 9-year old boy ${ }^{23,24}$. This potential danger, along with a possible correlation between initial symptom manifestation during early life and high disease severity ${ }^{16,25}$, further highlights the importance of early recognition of symptoms and timely diagnosis.

Recognition of prodromal symptoms in general is a cornerstone of C1-INH-HAE diagnosis and optimal disease management. Reshef et al. reported on the results of 3 surveys including a total of 113 patients with hereditary angioedema of which more than $90 \%$ with prodromal symptoms were able to predict an impending attack all or most of the time ${ }^{9}$.

The problem with erythema marginatum in particular is that, even though it is a common prodrome of C1-INH-HAE attacks in children, it is often mistaken for urticaria ${ }^{2,7,17,26}$ although, upon thorough examination, it can be easily distinguished from urticaria, which is pruritic, transient and more widespread, often consisting of smaller or larger elevated wheals ${ }^{26}$. While urticaria is treated with antiallergic agents and antihistamines, erythema marginatum, as a bradykinin-mediated phenomenon, does not respond to anti-allergic therapy. A further complication in the correct diagnosis of erythema marginatum is that, upon its presentation, even physicians who are aware of C1-INHHAE and its symptoms might exclude this diagnosis while wrongly assuming that the patient has urticaria, which was long believed to not occur in patients with C-INH$\mathrm{HAE}^{27}$. Misdiagnosis of erythema marginatum as urticaria might therefore contribute to diagnostic confusion and significantly delay the diagnosis of C1-INH-HAE, leading to ineffective and potentially harmful treatment regimens with anti-histamines, glucocorticoids and/or adrenaline ${ }^{26}$. Magerl et al. recently demonstrated a statistically significant longer diagnostic delay in C1-INH-HAE patients experiencing erythema marginatum than in patients without this prodrome ${ }^{10}$. Also, Rasmussen et al. showed that a high number of patients with hereditary angioedema had their erythema marginatum misdiagnosed as urticaria, which lead to a diagnostic delay that was 2 years longer than in other patients and also to inefficient treatment regimens with anti-allergic medications ${ }^{26}$. They further showed that urticaria can indeed occur in hereditary angioedema patients and thus must not lead to the exclusion of C1-INHHAE as a diagnosis. 26

The two patients described in our case report were young girls and also other reports indicate that erythema marginatum, as a prodrome of C1-INH-HAE, occurs more often in women than in men and more often in children and young adults than in older patients ${ }^{2,7,10}$. Therefore, a correlation of erythema marginatum and the hormonal profile of the patients, similar to the correlation of the severity of C1-INH-HAE with estrogen levels that has been reported previously ${ }^{28}$, might be possible but has not yet been investigated.

In line with our previously published case report, we want to emphasize the importance of adequate education of physicians with respect not only to C1-INH-HAE itself and to the availability of safe and efficacious treatment options for children, but also regarding correct symptom and prodrome recognition, especially in the pediatric population. Families with a history of C1-INH-HAE must be made aware of the hereditary nature of this disease and should consider testing all family members including newborns and children. Upon the occurrence of a non-pitting serpiginous rash, erythema marginatum can be suspected, thus allowing early intervention and adequate and timely treatment of the C1INH-HAE attack that might follow. 


\section{Contributors' statement}

I. Martinez-Saguer and H. Farkas drafted, critically reviewed and revised the manuscript and approved the final manuscript.

\section{Acknowledgements}

We Eva Kestner (Trilogy Writing \& Consulting GmbH, Germany) for medical writing services on behalf of CSL Behring GmbH.

\section{Conflict of interest}

I. Martinez-Saguer has received honoraria for lectures and funding for travel expenses from CSL Behring. She is a consultant for the following companies: Baxter, Bayer, CSL Behring, Octapharma, Shire and Sobi. Henriette Farkas has received consultancy/speaker fees and honoraria from Shire Human Genetic Therapies Inc., Swedish Orphan Biovitrum and CSL Behring.

\section{References}

1. Zanichelli A, Magerl M, Longhurst H, Fabien V, Maurer M. Hereditary angioedema with C1 inhibitor deficiency: delay in diagnosis in Europe. Allergy Asthma Clin Immunol. 2013; 9(1): 29.

2. Bygum A. Hereditary angio-oedema in Denmark: a nationwide survey. Br J Dermatol. 2009; 161(5): 1153-1158.

3. Martinez-Saguer I, Farkas H. Erythema Marginatum as an Early Symptom of Hereditary Angioedema: Case Report of 2 Newborns. Pediatrics. 2016; 137(2): 1-4.

4. Agostoni A, Aygören-Pürsün E, Binkley KE, et al. Hereditary and acquired angioedema: problems and progress: proceedings of the third C1 esterase inhibitor deficiency workshop and beyond. J Allergy Clin Immunol. 2004; 114(Suppl3): S51-131.

5. Banerji A, Busse P, Christiansen SC, et al. Current state of hereditary angioedema management: a patient survey. Allergy Asthma Proc. 2015; 36(3): 213-217.

6. Prematta MJ, Kemp JG, Gibbs JG, Mende C, Rhoads C, Craig TJ. Frequency, timing, and type of prodromal symptoms associated with hereditary angioedema attacks. Allergy Asthma Proc. 2009; 30(5): 506-511.

7. Farkas H, Harmat G, Fáy A, et al. Erythema marginatum preceding an acute oedematous attack of hereditary angioneurotic oedema. Acta Derm Venereol. 2001; 81(5): 376-377.

8. Starr JC, Brasher GW. Erythema marginatum preceding hereditary angioedema. J Allergy Clin Immunol. 1974; 53(6): 352-355.

9. Reshef A, Prematta MJ, Craig TJ. Signs and symptoms preceding acute attacks of hereditary angioedema: results of three recent surveys. Allergy Asthma Proc. 2013; 34(3): 261-266.

10. Magerl M, Doumoulakis G, Kalkounou I, et al. Characterization of prodromal symptoms in a large population of patients with hereditary angio-oedema. Clin Exp Dermatol. 2014; 39(3): 298-303.

11.Prematta MJ, Bewtra AK, Levy RJ, et al. Per-attack reporting of prodromal symptoms concurrent with C1-inhibitor treatment of hereditary angioedema attacks. Adv Ther. 2012; 29(10): 913-922.

12.0sler W. Hereditary angio-neurotic oedema. Am J Med Sci. 1888; 95 362-367.

13.Starr JC, Brasher GW, Rao A, Posey D. Erythema marginatum and hereditary angioedema. South Med J. 2004; 97(10): 948-950.

14.Wahn V, Aberer W, Eberl W, et al. Hereditary angioedema (HAE) in children and adolescents--a consensus on therapeutic strategies. Eur J Pediatr. 2012; 171(9): 1339-1348.

15.Craig T, Aygören Pürsün E, Bork K, et al. WAO guideline for the management of hereditary angioedema. World Allergy Organ J. 2012; 5(12): 182-199.

16.Farkas H. Pediatric hereditary angioedema due to C1-inhibitor deficiency. Allergy Asthma Clin Immunol. 2010; 6(1): 18.

17. Hubiche T, Boralevi F, Jouvencel P, Tä̈eb A, Leaute-Labreze C. [Reticular erythema signalling the onset of episodes of hereditary angioedema in a child]. Ann Dermatol Venereol. 2005; 132(3): 249-251.

18.Bork K, Staubach P, Eckardt AJ, Hardt J. Symptoms, course, and complications of abdominal attacks in hereditary angioedema due to C1 inhibitor deficiency. Am J Gastroenterol. 2006; 101(3): 619-627.

19.Zuraw BL. Clinical Practice. Hereditary angioedema. N Engl J Med. 2008; 359(10): 1027-1036.

20. Bennett G, Craig T. Hereditary angioedema with a focus on the child. Allergy Asthma Proc. 2015; 36(1): 70-73.

21.El-Hachem C, Amiour M, Guillot M, Laurent J. [Hereditary angioneurotic edema: a case report in a 3-year-old child]. Arch Pediatr. 2005; 12(8): $1232-1236$

22.0'Bier A, Muñiz AE, Foster RL. Hereditary angioedema presenting as epiglottitis. Pediatr Emerg Care. 2005; 21(1): 27-30.

23.Bork K, Hardt J, Witzke G. Fatal laryngeal attacks and mortality in hereditary angioedema due to C1-INH deficiency. J Allergy Clin Immunol. 2012; 130(3): 692-697.

24.Bork K, Siedlecki K, Bosch S, Schopf RE, Kreuz W. Asphyxiation by laryngeal edema in patients with hereditary angioedema. Mayo Clin Proc. 2000; 75(4): 349-354.

25. Martinez-Saguer I, Graff J, Rusicke E, et al. Does early clinical manifestation of hereditary angioedema (HAE) influence the clinical course of the disease? J Allergy Clin Immunol. 2013; 131(Suppl): AB30.

26. Rasmussen ER, de Freitas PV, Bygum A. Urticaria and Prodromal Symptoms Including Erythema Marginatum in Danish Patients with Hereditary Angioedema. Acta Derm Venereol. 2016; 96(3): 373-376.

27. Kanani A, Schellenberg R, Warrington R. Urticaria and angioedema. Allergy Asthma Clin Immunol. 2011; 7(Suppl1): S9.

28. Bouillet L, Longhurst H, Boccon-Gibod I, et al. Disease expression in women with hereditary angioedema. Am J Obstet Gynecol. 2008; 199: 484.e1-484.e4. 